\title{
Estimation of the flight thrust increment changing the operation mode of the engine
}

\author{
O.N. Korsun ${ }^{1, *}$, S.Yu. Prihodko ${ }^{2}$ and S.A. Sergeev ${ }^{1,3}$ \\ ${ }^{1}$ State Research Institute of Aviation Systems, Moscow, Russia \\ ${ }^{2}$ Moscow Aviation Institute (National Research University), Moscow, Russia \\ ${ }^{3}$ Moscow Automobile and Road Construction State Technical University, Moscow, Russia
}

\begin{abstract}
The paper presents a new method for flight test identification of the thrust increment when changing engine operation mode. It is known that the problem of separate estimation of the engine thrust and the aerodynamic drag force from the aircraft flight data belongs to a class of ill-posed. The idea of the presented method is to come out of the ill-posed class by changing the original problem formulation. To achieve this purpose we give up the absolute values of the thrust and proceed to estimating the thrust increment resulting from the change of the aircraft engine operation mode. The accuracy of the solution is improved by introducing the special flight test maneuver. The algorithm requires only a few on-board measured parameters, such as longitudinal and normal overload, airspeed and altitude, and angle of attack. The complimentary use of the engine gas-dynamic model may improve the estimates but is not obligatory. The proposed method also does not require a-priory estimates of the aerodynamic drag force.
\end{abstract}

\section{Introduction}

The paper presents a new method for flight test identification of the thrust increment when changing engine operation mode. It is known that the problem of separate estimation of the engine thrust and the aerodynamic drag force from the aircraft flight test data belongs to a class of ill-posed [1-3], in view of the collinearity of thrust and drag vectors at low angles of attack. There are several ways to deal with the problem, and all of them are their own constraints. The simplest way is to take one of above mentioned collinear vectors for known. Indeed, when drag is postulated the thrust may be estimated easily from flight data and vice versa $[4,5]$. Another variant is to insert special load sensors into the engine mounting system [6]. The most widespread approach is to calculate thrust using the engine gas-dynamic model and pressure and temperature measurements in the key sections of the flow [7]. The paper [8] discusses a method for drag and thrust absolute value separate identification from flight test data based on a specially designed test maneuver.

The idea of the method presented in this article is to extract the problem out of the ill-posed class by changing the original formulation. To achieve this purpose we give up the absolute values of the thrust and proceed to estimating the thrust increment resulting from the change of the aircraft engine operational mode. The new problem is correct from the mathematical standpoint. Moreover we may improve the accuracy of the solution by introducing the specific flight test maneuver. The idea of this maneuver is to compensate the change of the thrust by gravity force using dive or climb. In this case the principal flight parameters affecting the engine thrust, such as Mach number and angle of attack, keep constant with high precision, and the only parameter of significant variation is the engine operation mode. It allows us to estimate the corresponding increment of thrust. The duration of this test maneuver should not exceed $30 \ldots 40$ $\mathrm{s}$, so the change of the altitude is not to be significant.

The detailed description is given further in the next part of the paper.

\section{Flight test manoeuvres performance}

The flight experiment is carried out as follows. As a first step the aircraft is balanced in straight and level flight at constant altitude, air velocity and angle of attack $\alpha$ (Fig.1).

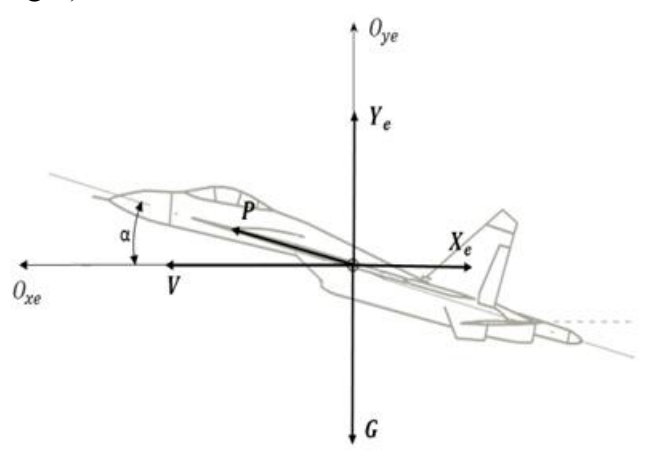

Fig. 1. Aircraft balanced motion in straight and level flight. 
Then the pilot carries out the control stick stepwise symmetric deviations in pitch channel. It is required that the air velocity should be approximately constant and the fluctuations of the angle of attack amounted to $\pm(2 \ldots 3)$ degrees.

Next the pilot changes the engine operation mode, for example, to increase the thrust, and proceeds to straight climb in order to keep the air velocity and angle of attack

approximately constant. The increased thrust is compensated by the climb, that is by the projection of the gravity force (Fig. 2).

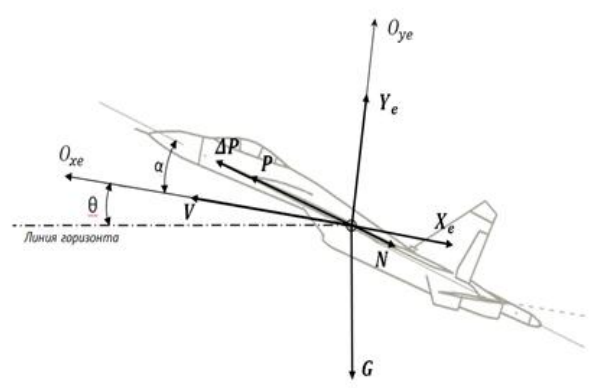

Fig. 2. Straight climb with constant velocity.

During the straight climb it is recommended to repeat the control stick stepwise symmetric deviations in pitch channel, but this is not obligatory. Next the pilot changes the engine operation mode, for example, to diminish the thrust, and proceeds to straight dive in order to keep the air velocity and angle of attack approximately constant. So, the dive is symmetric to the climb. One may chose several fixed engine modes. It enables to cover the full range of thrust at given Mach number and altitude.

In order to estimate all the admissible regimes we should choose Mach and altitude nodes through all the flight performance envelope of the aircraft.

Since the duration of this maneuver is small the altitude variations are limited and commonly do not affect the functioning of the engine.

It was confirmed that a qualified professional pilot performs testing regimes like that quite easily.

The example of pitch angle variation for a training aircraft is presented Fig. 3 .

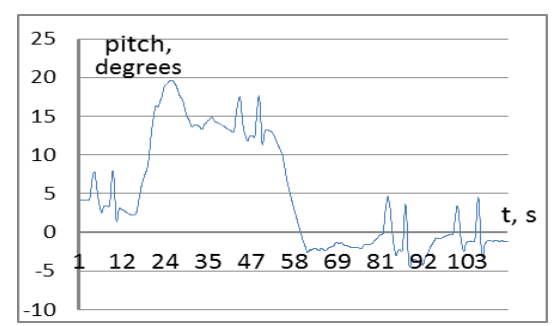

Fig. 3. The pitch angle variation during the flight test maneuver.

\section{Formulae for estimating the thrust increment}

Let us introduce the model of the process which includes the parabolic approximation of the drag force and considers the thrust as a vector sum of the input and output impulses. It is assumed that the input impulse $P_{\text {in }}$ is directed along the axis $O x_{e}$ of the air velocity coordinate system, that is coincides with the direction of the air velocity vector. The output impulse $P_{\text {out }}$ is directed along the engine axis, which is commonly deviated from the axis $O x$, associated with the aircraft, by the engine installation angle $\phi_{i n s t}$. The $O x_{e}$ axis force projections form the following equation

$$
\begin{aligned}
& m g n_{x_{e}}(t)=-q S c_{x_{o}}-q S c_{x}^{\alpha} \alpha(t)- \\
& -q S c_{x}^{\alpha^{2}} \alpha^{2}(t)-P_{\text {in }}+P_{\text {out }} \cos \left(\phi_{\text {inst }}+\alpha(t)\right),
\end{aligned}
$$

where $n_{x_{e}}(t)$ is the overload projection along the $O x_{e}$ axis; $S$ - equivalent wing area; $m$ - aircraft mass; $g-$ gravity force acceleration; $q-$ the dynamic pressure; $\alpha(t)$ - angle of attack; $c_{x_{o}}, c_{x}^{\alpha}, c_{x}^{\alpha^{2}}$ - parameters for the drag force parabolic approximation.

To transform (1) the following trigonometric formula is used:

$$
\cos \left(\phi_{\text {inst }}+\alpha\right)=\cos \phi_{\text {inst }} \cos \alpha-\sin \phi_{\text {inst }} \sin \alpha .
$$

In the vicinity of zero angles of attack we get

$$
\cos \alpha \approx 1-\frac{1}{2} \alpha^{2} ; \sin \alpha \approx \alpha .
$$

Then at small angles of attack the equation (1) for force projections is:

$$
\begin{aligned}
& m g n_{x_{e}}(t)=-q S c_{x_{o}}-q S c_{x}^{\alpha} \alpha(t)-q S c_{x}^{\alpha^{2}} \alpha^{2}(t)-P_{i n}+ \\
& +P_{\text {out }} \cos \phi_{\text {inst }}-\frac{1}{2} P_{\text {out }} \alpha^{2}(t) \cos \phi_{\text {inst }}-P_{\text {out }} \alpha(t) \sin \phi_{\text {inst }}
\end{aligned}
$$

Let us write down the last equation for the initial stage of the flight test regime, that is straight and level flight. Let us also denominate input and output impulses for this initial stage $P_{i n_{0}}$ and $P_{o u t_{0}}$ and regroup the equation's right part :

$$
\begin{aligned}
& m g n_{x e}(t)=1 \cdot\left(-q S c_{x_{o}}+P_{o u t_{0}} \cos \phi_{i n s t}-P_{i n_{0}}\right)- \\
& -\alpha(t)\left(q S c_{x}^{\alpha}+P_{o u t_{0}} \sin \phi_{\text {inst }}\right)- \\
& -\alpha^{2}(t)\left(q S c_{x}^{\alpha^{2}}+\frac{1}{2} P_{o u t_{0}} \cos \phi_{\text {inst }}\right) .
\end{aligned}
$$

Taking into account that the sum of the input $P_{i n}$ and output $P_{\text {out }}$ impulses form the so-called effective thrust $P_{\text {eff }}[7,8]$ we obtain:

$$
P_{\text {eff }}=-P_{\text {in }}+P_{\text {out }} \cos \phi_{\text {inst }}
$$

Let us substitute (4) into equation (3):

$$
\begin{aligned}
& m g n_{x e}(t)==1 \cdot\left(-q S c_{x_{0}}+P_{\text {effo }}\right)-\alpha(t)\left(q S c_{x}^{\alpha}+P_{\text {out }} \sin \phi_{\text {inst }}\right)- \\
& -\alpha^{2}(t)\left(q S c_{x}^{\alpha^{2}}+\frac{1}{2} P_{\text {out }} \cos \phi_{\text {inst }}\right)=a_{0}+\alpha a_{1}+\alpha^{2} a_{2} .
\end{aligned}
$$

The equation (5) corresponds to the flight test initial stage which includes the straight level flight and control stick stepwise symmetric deviations in pitch channel. It is clear that the equation (5) fits the common multiple regression model with factors $1, \alpha(t), \alpha^{2}(t)$. The angle of attack $\alpha$ and overload $n_{x e}$ may be taken from onboard measurements. So, out of the stepwise symmetric 
deviations interval we may obtain the following parameter estimates using the least square multiple regression:

$$
\begin{aligned}
& \widehat{a}_{0}=\left(-q S c_{x_{0}}+P_{e f f_{0}}\right) ; \\
& \widehat{a}_{1}=\left(q S c_{x}^{\alpha}+P_{\text {out }} \sin \phi_{\text {inst }}\right) ; \\
& \widehat{a}_{2}=\left(q S c_{x}^{\alpha^{2}}+\frac{1}{2} P_{\text {out }} \cos \phi_{\text {inst }}\right) .
\end{aligned}
$$

Now to provide identification of the thrust increment we are to write the equation similar to (5) for time interval after the change of the engine operational mode. In this case we introduce that the input $P_{i n}$ and output $P_{\text {out }}$ impulses are dependent of time:

$$
\begin{gathered}
m g n_{x e}(t)=1 \cdot\left(-q S c_{x_{0}}+P_{e f f}(t)\right)- \\
-\alpha(t)\left(q S c_{x}^{\alpha}+P_{\text {out }}(t) \sin \phi_{\text {inst }}\right)- \\
-\alpha^{2}(t)\left(q S c_{x}^{\alpha^{2}}+\frac{1}{2} P_{\text {out }}(t) \cos \phi_{\text {inst }}\right) .
\end{gathered}
$$

Finally, we may multiply the estimates (6) by proper factors $1, \alpha(t), \alpha^{2}(t)$ and subtract them from the equation (7). Thus we get the formula for estimating the the effective thrust increment:

$$
\begin{aligned}
& \Delta P_{\text {eff }}(t)=m g n_{x_{e}}(t)-\hat{a}_{0}+\hat{a}_{1} \alpha(t)+\hat{a}_{2} \alpha(t)^{2}+ \\
& +\Delta P_{\text {out }}(t)\left(\alpha \sin \phi_{\text {inst }}+\alpha^{2} \frac{1}{2} \cos \phi_{\text {inst }}\right) .
\end{aligned}
$$

The last term in the (8) right part is a systematic error. It is not considerable at small angles of attack and small $\phi_{\text {inst }}$. Additionally, to reduce this error the estimates of $\Delta P_{\text {out }}$, obtained from the engine gasdynamic model, should be used. Errors of this model do not matter here, because of smallness of the whole term.

Effect of random errors can be significantly reduced by using more sophisticated identification methods [2,3] instead of the multiple regression. Systematic error of measurement of angles of attack and airspeed should be excluded at the stage of flight tests, using known identification techniques [2, 3].

Figure 4 shows the effective thrust increment estimates obtained by the proposed method (red line), and the values of the increments at the same flight regime, calculated independently from the engine gasdynamic model described in [7]. The good enough coincidence of two unrelated assessments confirms performance of the proposed method.

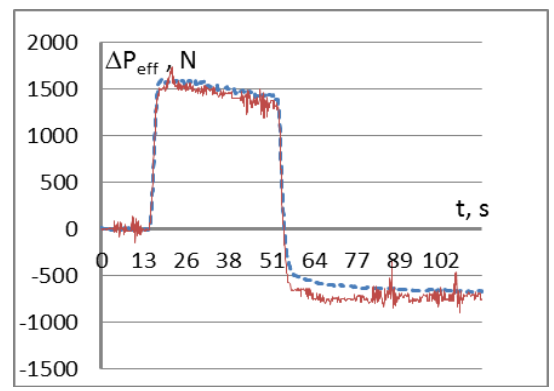

Fig. 4. Estimates increments effective (the proposed method) and internal thrust engines (model, made according to bench tests) on a test mode in flight

\section{Conclusions}

1. The proposed method improves the accuracy and stability of the thrust estimation by switching to mathematically correct problem formulation instead of ill-posed .

2. If necessary, to determine the absolute value of thrust, this method should be used in conjunction with a method of estimating the absolute value of thrust for the given Mach number and altitude [8].

3. The proposed method may be used for purposes of monitoring the engines technical state since the thrust increments at fixed changes in engine operating mode are strongly correlated with the deterioration of the engine.

This work is supported by Russian Foundation for Basic Research (RFBR), project 18-08-00921-a.

\section{References}

1. A.N. Tikhonov, V.Y. Arsenin, Methods for incorrect problems solution (Moscow, Nauka, 1986) In Russian

2. R.V. Jategaonkar, Flight vehicle system identification: a time domain methodology (American Institute of Aeronautics and Astronautics, Reston, 2006)

3. V. Klein, E.A. Morelli, Aircraft system identification: theory and practice (American Institute of Aeronautics and Astronautics, Reston, 2006)

4. G.Girija, V. Parameswaran, JR. Raol, S. Srinathkumar, Journal of Aeronautical Society of India, 43 (4), 285-292 (1991)

5. Hoff J.C., Barbarose J.R. Application of a Stochastic In-Flight Thrust Determination Process to Real Engine Data, 3rd CTA DLR Workshop on Data Analysis and Flight Control (S.J. Campos, Brasil, 2009)

6. Muhammad H. Muhardi, Kuntjoro W., Sritjiono B.E. In-Flight Thrust Determination by Load Meassurement on the Engine Mounting System, ICAS- 2000 Congress, pp. 533.1-533.7 (2000)

7. O.N. Korsun, I.A. Leshchenko, M. Nemichev, Mechatronics, automation control, 11, 50-54 (2011) [In Russian]

8. O.N. Korsun, B.K. Poplavsky, S.Ju. Prihodko, Procedia Computer Science, 103, 82-87 (2017) 